Child Neuropsychology Vol. 3(1):13-27 (1997)

ISSN: (print 0929-7049)(online 1744-4136)

doi: $10.1080 / 09297049708401365$

This is a peer reviewed pre-print version of the following article from Child Neuropsychology: Attention

Performance in an Epidemiological Sample of Urban Children: The Role of Gender and Verbal Intelligence, which

has been pulished in final form at:

http://www.psypress.com

http://www.tandf.co.uk/journals/titles/09297049.asp

http://www.tandfonline.com/doi/abs/10.1080/09297049708401365

(C) 1997 Taylor \& Francis

\title{
Attention Performance in an Epidemiological Sample of Urban Children: The Role of Gender and Verbal Intelligence
}

\author{
Daisy M. Pascualvaca, Section on Clinical and Experimental Neuropsychology, \\ Laboratory of Psychology and Psychopathology, National Institute of Mental \\ Health, Bruno J. Anthony, Section on Clinical and Experimental Neuropsychology, \\ Laboratory of Psychology and Psychopathology, National Institute of Mental \\ Health, and the Department of Psychiatry, University of Maryland School of \\ Medicine, L. Eugene Arnold, Child and Adolescent Disorders Research Branch, \\ National Institute of Mental Health, George W. Rebok, Prevention Research Center, \\ Department of Mental Hygiene, School of Hygiene and Public Health, Johns \\ Hopkins University, Mary Beth Ahearn, Prevention Research Center, Department \\ of Mental Hygiene, School of Hygiene and Public Health, Johns Hopkins University, \\ Sheppard G. Kellam, Prevention Research Center, Department of Mental Hygiene, \\ School of Hygiene and Public Health, Johns Hopkins University, Allan F. Mirsky, \\ Section on Clinical and Experimental Neuropsychology, Laboratory of Psychology \\ and Psychopathology, National Institute of Mental Health
}

\begin{abstract}
We administered a comprehensive attentional battery to an epidemiologically defined sample of 435 firstand second-grade children to assess the influence of gender and verbal intelligence on attention. The battery included three versions of the continuous performance test (CPT), two digit cancellation tasks, three subtests from the WISC-R, and the Wisconsin Card Sorting Test. The results indicated that both gender and intelligence had an impact on attentional performance. Girls performed better than boys; they made fewer errors on the CPT and obtained higher scores on the digit cancellation task and the Coding subtest of the WISC-R. Children with higher verbal intelligence also performed better on the attentional tests, but this advantage was not observed across measures or levels of performance. For example, children with limited verbal skills performed significantly worse than their peers only in measures with high processing demands (the degraded CPT and the distraction version of the digit cancellation task).
\end{abstract}

Problems with attention represent one of the most pervasive behavioral disturbances encountered in psychiatric and educational contexts, and are estimated to affect as many as 30\% of all school-aged children (Cantwell, 1975; Kellam, Branch, Agrawal, \& Ensminger 1975; Offord et al., 1987; Rutter, Tizard, \& Whitmore, 1970). Despite the ubiquity of attention deficits and their detrimental effects on the child's functioning, little is known about the factors that influence attentional performance in normal children. There is little information, for example, regarding the impact of certain characteristics such as age, intelligence, and gender, or environmental factors such as family background and socioeconomic status, on attentional performance. In this study, we report on the role of two of these characteristics, intelligence and 
gender; the impact of other factors such as ethnic background, socioeconomic status, and family composition will be presented in a separate communication.

There is considerable evidence to indicate that age influences attentional performance. For example, the capacity to sustain attention, inhibit inappropriate responses, and shift attentional set all improve during childhood (Chelune \& Baer, 1986; Gale \& Lynn, 1972; Greenberg \& Waldman, 1993; Halperin, Sharma, Greenblatt, \& Schwartz, 1991; Levy, 1980). The importance of other characteristics such as intelligence and gender is less clear. Several studies have found no association between attention and intelligence in children with intact cognitive capacities (Gale \& Lynn, 1972; Halperin et al., 1991). However, children with mental retardation and borderline intelligence have more difficulty than their peers in sustaining a focus of attention (Carter \& Swanson, 1995; see Warm \& Berch, 1985 for a review) and ignoring distracting information (Hagen \& Huntsman, 1971). There are several possibilities for the discrepancy in these findings. It is possible, for example, that the relationship between attention and intelligence is not uniform across the range of abilities or varies according to the specific attention process. It is also possible that specific conditions or task requirements have a different impact at various levels of intelligence. Unfortunately, these issues cannot be addressed with the available data because most studies have either used a single objective measure of attention or have only studied children within a narrow range of cognitive abilities.

The same limitations apply to the investigation of gender differences in attentional capacities. Most studies of attentional performance in children have not focused on gender differences, and the few studies that are available have used only one measure. Some studies have found no differences between boys and girls in attention tests such as the Attentional Capacity Test (Weber \& Segalowitz, 1990) whereas studies using the Continuous Performance Test have yielded inconsistent results (Greenberg \& Waldman, 1993; Kirchner \& Knopf, 1974; Levy \& Hobbes, 1979). Since these tests measure specific components of attention, the results cannot be generalized to other attention processes. Furthermore, evidence from various lines of investigation suggests that boys and girls may differ in their attention functions. For example, many disorders that are characterized by attention problems, such as Attention-Deficit/Hyperactivity Disorder (AD/HD), are not only more commonly diagnosed among boys (American Psychiatric Association, 1994), but are expressed differently between the genders (Berry, Shaywitz, \& Shaywitz, 1985; Brown, Madan-Swain, \& Baldwin, 1991; James \& Taylor, 1990). In addition, the symptoms associated with impaired attention appear to vary depending on the child's gender. Studies of nonclinical samples report that attention problems are associated with behavioral difficulties (Halperin et al., 1988; Kellam et al., 1975; Kellam et al., 1991; McGee, Williams, \& Silva, 1985; Rebok, Hawkins, Krener, Mayer, \& Kellam, 1996) and lead to poor academic achievement (Kellam et al., 1991; Rowe \& Rowe, 1992) among both boys and girls, but to depression only among girls (Kellam et al., 1991). Therefore, understanding the role of gender on attentional performance may help us (1) differentiate normal gender differences from variations in the manifestation of disorders characterized by impaired attention and (2) clarify the reasons for the higher vulnerability of boys to these disorders.

We view attention as a multidimensional construct and selected our measures to assess conceptually different aspects of attention based on the research conducted by Mirsky and colleagues (Mirsky, 1987; Mirsky, Anthony, Duncan, Ahearn, \& Kellam, 1991). Distilling the large amount of data on attention from cognitive psychology. neuropsychology, and psychopathology, these investigators suggested that various attention measures reflect different cognitive processes. Based on the results of a factor analysis of a battery of neuropsychological 
tests, these authors identified four separate processes or elements including the ability to focus; the capacity to maintain or sustain a focus over time; the ability to change or shift attentive focus in a flexible and adaptive manner; and the attention required to encode information (e.g., recode, recall, and reorder) once initial registration has occurred (Mirsky et al., 1991). These investigators identified this factor structure initially in a sample of 203 adults and subsequently replicated the findings using the present sample (Mirsky et al., 1991). For the current study, we included the measures employed by these investigators, and added other measures to assess aspects of attention particularly relevant to classroom activity; these include the ability to resist distracting events and the capacity to control the intensity of attentive effort.

The children in this study came from varied backgrounds and were not chosen on the basis of having teacher- or parent-identified attention problems, but rather were representative of the population at large. A multi-stage sampling and assessment strategy was employed in selecting the present sample from a larger first-stage sample (Kellam \& Rebok, 1992). A first-stage sample is an entire cohort of children that is representative of a defined population. The sample described here is referred to as a second-stage sample. This sample is smaller yet representative of the original population, and is suitable for in-depth investigation. This sample is particularly relevant for the study of attentional capacities because many of the children came from urban areas associated with a high risk of attention and behavior problems.

These children are participating in a longitudinal study conducted by the Prevention Research Center of the Johns Hopkins University. The aim of the study is to identify antecedents of maladaptive behaviors and to evaluate the effects of two classroom-based intervention strategies. The study examined the effects of two classroom-based preventive interventions designed to improve academic achievement and reduce maladaptive aggressive and shy behaviors (Dolan et al., 1993). There were virtually no effects of intervention on attention performance in the sample of 435 children, and no significant interactions between intervention status and gender or intelligence. We report here the results of the initial assessment, completed when the children were enrolled in the second grade. The composition of the sample and the nature of the assessment procedures give us a unique opportunity to study individual differences in attentional performance in a large group of children, eliminating possible referral and selection biases. As indicated previously, our objective was to examine the influence of gender and intelligence on various aspects of attention and, more specifically, to address the following questions:

(1) Will an unselected, non clinical sample of boys and girls differ in their attention capacities?

(2) What is the relationship between gender differences in attentional performance, if any, and boys' increased vulnerability to attention disorders?

(3) What is the relationship between intelligence and various attention elements?

\section{Methods}

\section{Subjects}

The sample consisted of 435 children selected from an original cohort of first graders who participated in a collaborative study by the Prevention Research Center of the Johns Hopkins University and the Baltimore City Public Schools. The original cohort came from diverse socioeconomic backgrounds and included all children entering the first grade of 19 public schools in Baltimore in the Fall of 1985. The participating schools were selected after a review of East Baltimore neighborhood characteristics with the Baltimore City Department of 
Planning. In this review, five urban areas that differed in terms of income level, ethnicity, family composition, and type of housing were chosen. Urban area 1 was a predominantly Caucasian area in which married couples with incomes in the low-to middle range resided in well-maintained row houses. Urban area 2 comprised large public housing projects and was mostly African American in composition. Urban area 3 was populated by middle-income African American families who resided in well-maintained row houses. Urban area 4 was an integrated area, with middle-income married couples living in detached houses. Urban area 5 was a Caucasian area, comprised of moderate-income married couples who resided in detached or semidetached homes. Within each urban area, three or four similar schools matched for achievement scores, ethnicity, and socioeconomic status were identified.

In the Spring of 1987, 743 children from the original sample (most in second grade) had parental consent and teacher ratings of classroom behavior and were, therefore, eligible to participate in the study. Because of time limitations, the entire cohort could not be evaluated. In order to preserve the representative nature of the population, sampling was carried out with a replicate sampling scheme, grouping the 19 schools into three replicates of schools matched for intervention status and urban area. From March to June of 1987, testing was completed for all children in replicates one and two, and a portion in replicate three. One third of the students in each of the five schools in replicate three (77 of 230) were selected randomly to remain in the sampling frame. Within the three replicates, 475 assessments were attempted and 435, or 91.6\%, were completed. Forty children were not assessed because of absences, transfers, and school scheduling conflicts.

The study sample included 214 boys and 221 girls, most between the ages of 7.5 and 8 years (mean $=7.9$ years). The sample was $63.7 \%$ African American, 35.3\% Caucasian, and also included 1 Asian American, 3 Native Americans, and 1 Hispanic child. Most children $(n=380)$ were enrolled in second grade; 55 children ( 24 boys and 31 girls), or almost $13 \%$ of the sample, were repeating the first grade. Chi-square comparisons between the study sample and the remainder of the original cohort did not yield any differences in gender or race between the two groups.

\section{Measures}

Table 1 presents a list of the attention measures included in the study in the order in which they were administered. This order was chosen to minimize the effects of fatigue and boredom. As previously indicated, our selection of measures was based on the model developed by Mirsky and colleagues. In previous publications, some of us found that these measures group into separate factors and proposed that they assess distinct attentional elements (Mirsky, 1987; Mirsky et al., 1991). More specifically, the Digit Cancellation task and the Coding subtest of the WISC-R were found to assess the child's ability to focus on a particular stimulus and make a rapid response. The Continuous Performance Test (CPT) was considered to be a measure of sustained attention whereas the Wisconsin Card Sorting Test tapped the capacity to shift the focus of attention adaptively. Finally, the Arithmetic and Digit Span subtests of the WISC-R measure the facility to encode information once initial registration had occurred.

In addition to the attention tests, we included the Peabody Picture Vocabulary Test-Revised (Dunn \& Dunn, 1981) as an estimate of verbal intelligence. A description of these tests follows.

Continuous Performance Tests (CPT) 
We administered three versions of the Continuous Performance Test (Rosvold, Mirsky, Sarason, Bransome, \& Beck, 1956) using a Sunrise Systems Yankee II computer and a stimulus display with a 2 in. $x 2$ in. screen. In all CPT versions, children saw individual letters presented on the screen at a rate of one per second, and were instructed to press a response button as quickly as possible whenever the letter ' $\mathrm{X}$ ' appeared. For each task, a total of 300 letters was presented with a 100-ms stimulus duration and a 900-ms interstimulus interval. Targets (Xs) were presented in a pseudorandom sequence with a probability of .20. Each CPT version included 60 randomly presented targets and lasted approximately $6 \mathrm{~min}$.

\begin{tabular}{l}
\hline Name of Instrument \\
Continuous Performance Test: Standard version \\
Digit Cancellation: Standard condition \\
Digit Cancellation: Distraction condition \\
Continuous Performance Test: Auditory distraction version \\
Wechsler Intelligence Scale for Children-Revised (WISC-R) \\
$\quad$ Digit span subtest \\
$\quad$ Arithmetic subtest \\
Continuous Performance Test: Degraded version \\
Wisconsin Card Sorting Test (WCST)
\end{tabular}

Table 1. Attention Battery.

The three CPT versions varied in terms of stimulus presentation and processing demands. In the Standard version, the children were instructed to press the response button whenever they saw an ' $\mathrm{X}$ ' on the screen. The Degraded CPT was similar to the Standard version except that the letters were blurred by placing a piece of ground glass over the display screen. In the Distraction task, children heard a different sequence of letters delivered over earphones at the same rate as the visually presented letters. They were instructed to ignore the aurally presented letters and to respond to the visual stimuli. All children received practice trials in each task prior to beginning testing.

In each version, we recorded the total number of correct responses (hits), omission errors (misses). commission errors (false alarms), and reaction time for correct responses. This information was available for the first half (i.e., initial 30 targets) and second half (i.e., second sequence of 30 targets) of each CPT.

\section{Digit Cancel lotion Task}

The Digit Cancellation task was modelled after the distractibility test described by Lifshitz, Kugelmass, and Karov (1985). Two conditions were included in the present study. In the Standard condition, children were shown an $81 / 2$ in. $x 11$ in. sheet of paper with a $15 \mathrm{~cm} \mathrm{x}$ $10 \mathrm{~cm}$ array of numbers and were instructed to scan each row of the array as quickly as possible from left to right and cross out the numbers 3 and 7. In the Distraction condition, children heard digits presented through earphones at a rate of 1 per s while they were scanning 
the array. They were instructed to cross out the numbers 2 and 4 in the array and to ignore the auditory digits. Each task yielded three scores: time to complete the task, number of targets missed (omission errors), and number of incorrect responses (commission errors).

\section{Wechsler Intelligence Scale for Children-Revised (WISC-R) (Wechsler, 1974)}

The Arithmetic, Coding, and Digit Span subtests of the WISC-R were included as additional measures of attention. These subtests constitute the freedom-from-distractibility factor described by Kaufman $(1975,1979)$.

Wisconsin Card Sorting Test (WCST) (Heaton, 1981)

The WCST is widely used to measure concept formation and the ability to shift set. In this task, the child is given a set of cards and asked to place each card under a four-choice display which differ in terms of color, form, or number. The child is not given further instructions but receives feedback ('right' or 'wrong') after each card placement. After 10 correct responses in one category, the category is changed without informing the subject. In agreement with standard instructions, the task was discontinued when the child completed six categories (sorted the cards using each category twice) or had sorted all 128 cards. We also discontinued the task if a child had not completed any categories after sorting 64 cards. Number of correct responses, perseverative errors, nonperseverative errors, and failures to maintain set were used as dependent measures.

Peabody Picture Vocabulary Test-Revised, Form L (PPVT-R) (Dunn \& Dunn, 1981)

The PPVT-R is a test of receptive vocabulary that correlates highly with standardized intelligence tests and was included in this study as an estimate of verbal intelligence. In this task, the examiner says a word and the child has to point to one picture among a set of four alternatives that depicts the word.

\section{Procedure}

Children were tested individually in their school during regular hours by four examiners trained in the administration of the instruments. The battery of tests took approximately $70 \mathrm{~min}$ to complete. All children were tested within a 3-month period.

\section{Results}

\section{Gender Differences}

A series of ANCOVAs controlling for age were performed to compare boys and girls on the attention measures. Girls outperformed boys on the Digit Cancellation task, where they had faster completion times and fewer errors of omission $(p<.02)$. Although the same findings were obtained in the Standard and Distraction conditions, there was a significant interaction between gender and version of the Digit Cancellation task $(p<.001)$. This interaction accentuated gender differences in the Distraction condition relative to the Standard condition. In other words, introducing the distraction slowed the performance of boys more than that of girls and also resulted in relatively more omission errors (Table 2). Girls also obtained higher scores on the Coding subtest of the WISC-R. This finding provided further support for the superiority of girls at focusing attention on a particular stimulus, ignoring irrelevant stimuli, and making a rapid response.

The CPT results shown in Table 2 also indicated gender differences in response time, in 
this case favoring boys. Boys had shorter reaction times to target stimuli in all versions of the CPT $(p<.02)$ and responded, on the average, $12-18 \mathrm{~ms}$ faster than girls. Boys also made more commission errors in all versions of the CPT, but did not differ from girls in the number of correct responses $(p>.05)$. Thus, unlike the performance of girls on the Digit Cancellation task, boys tended to sacrifice accuracy for speed in the CPT

Because boys had faster reaction times and were more likely to respond incorrectly on the CPT, we examined gender differences in speed-accuracy trade-offs by correlating reaction time with number of commission errors. These analyses revealed that reaction time was negatively correlated with commission errors only in the Degraded task $(r=-.30$ for boys, -.33 for girls; $p<.0001)$. Unexpectedly, the correlations between reaction time and number of errors in the Standard and Distraction versions were nonsignificant for boys, and positive for girls. That is, for girls, slower reaction times were associated with a larger number of errors in both the Standard $(r=.27, p<.0001)$ and Distraction $(r=.38, p<.0001)$ versions.

We then tested possible gender differences in response strategy using signal detection parameters. We selected the nonparametric indices of sensitivity, $\alpha^{\prime}$, and response criterion, $\beta^{\prime}$, instead of their parametric counterparts $\left(\mathrm{d}^{\prime}\right.$ and $\beta$ ) because of the lack of normality in some of our distributions (Grier, 1971). Our results indicated that boys and girls obtained similar $\alpha^{\prime}$ values in all CPTs $(p>.10)$. Differences in $\beta^{\prime}$ reached significance only in the Standard version, with boys obtaining lower scores. Therefore, we obtained only partial support with this metric for the hypothesis that boys adopt a more lenient response criterion.

There were no other gender differences in CPT performance. Comparisons of the children's scores in the first and second halves of the CPT did not reveal any significant main effect of time (i.e., first vs. second half of the task) or significant interactions between time and gender. Similarly, the Degraded version was more difficult than the Standard and Distraction version, but there was no significant interaction between task version and gender. These findings indicate that boys and girls are equally proficient at sustaining a focus of attention over time, although boys tend to have more difficulty withholding erroneous responses.

Boys and girls did not differ in their capacity to shift their focus of attention, as reflected by their performance on the WCST $(p>.10)$. They also obtained similar scores on the Arithmetic and Digit Span subtests of the WISC-R, suggesting a comparable ability to encode numerical information.

\section{Verbal Intelligence}

We examined first the influence of the child's verbal intelligence on attentional performance through a correlation matrix using age as a covariate. Table 3 displays the values of these correlations as well as the results of the Fisher $\mathrm{Z}$ transformation used to compute differences in the magnitude of the correlations between boys and girls. Before reviewing the specific findings, we would like to emphasize that, although the magnitudes of some the significant correlations are small on an absolute level, these values are of a substantial size for epidemiological samples.

As can be seen in Table 3, higher verbal intelligence was associated with better attentional performance for both boys and girls, although this association was generally stronger for boys. For example, number of errors in the CPT was negatively correlated with PPVT-R scores for both genders, but the correlations were consistently significant only for boys. For boys, performance on the PPVT-R was also negatively correlated with completion time on the Distraction condition of the Digit Cancellation test. In fact, all measures that assess the child's ability to process information rapidly (e.g., CPT reaction time, completion time in the Digit 
Cancellation tasks, and scores in the Coding subtest) tended to be negatively correlated with PPVT-R

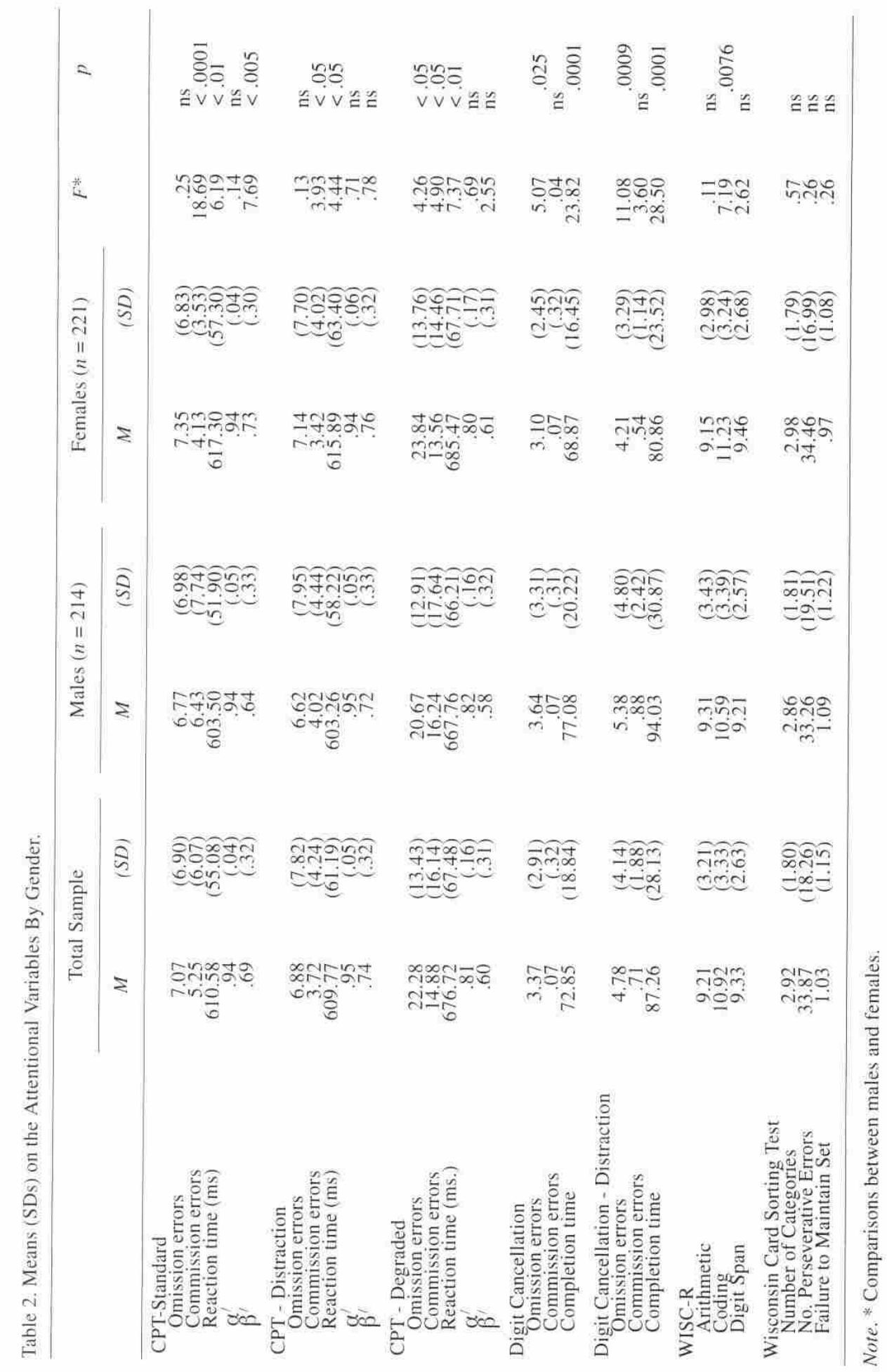




\begin{tabular}{|c|c|c|}
\hline & \multicolumn{2}{|c|}{ PPVT-R Standard Scores } \\
\hline & Girls & Boys \\
\hline \multicolumn{3}{|l|}{ CPT-Standard } \\
\hline Omission errors & -.11 & $-.24^{b, c}$ \\
\hline Commission errors & -.14 & $-.18^{\mathrm{a}}$ \\
\hline Reaction time & .01 & $-.20^{\mathrm{a}, \mathrm{c}}$ \\
\hline \multicolumn{3}{|l|}{ CPT-Distraction } \\
\hline Omission errors & -.15 & $-.20^{\mathrm{a}}$ \\
\hline Commission errors & $-.26^{\mathrm{b}}$ & $-.23^{\mathrm{b}}$ \\
\hline Reaction time & & $-.19^{\mathrm{a}}$ \\
\hline \multicolumn{3}{|l|}{ CPT-Degraded } \\
\hline Omission errors & $-.18^{\mathrm{a}}$ & $-.31^{b, c}$ \\
\hline Commission errors & $-.20^{\mathrm{a}}$ & $-.26^{\mathrm{b}}$ \\
\hline Reaction Time & .02 & -.07 \\
\hline \multicolumn{3}{|c|}{ Digit Cancellation - Standard } \\
\hline Omission errors & $-.20^{\mathrm{a}}$ & -.13 \\
\hline Commission errors & -.15 & .04 \\
\hline Completion time & -.01 & -.13 \\
\hline \multicolumn{3}{|c|}{ Digit Cancellation - Distraction } \\
\hline Omission errors & $-.20^{\mathrm{a}}$ & -.15 \\
\hline Commission errors & .10 & -.10 \\
\hline Completion time & .03 & $-.27^{\mathrm{b}, \mathrm{c}}$ \\
\hline \multicolumn{3}{|l|}{ WISC-R (raw scores) } \\
\hline Arithmetic subtest* & $.36^{\mathrm{b}}$ & $.34^{\mathrm{b}}$ \\
\hline Coding subtest* & -.09 & $.28^{\mathrm{b}, \mathrm{c}}$ \\
\hline Digit Span subtest* & $.18^{\mathrm{a}}$ & $.26^{\mathrm{b}}$ \\
\hline \multicolumn{3}{|l|}{ WCST } \\
\hline Categories* & $.31^{\mathrm{b}}$ & $.26^{\mathrm{b}}$ \\
\hline Perseverative Errors & -.16 & -.10 \\
\hline
\end{tabular}

* Positive correlations for these measures have the same meaning as negative correlations for the rest of the measures.

${ }^{\mathrm{a}} p<.01$;

${ }^{\mathrm{b}} p<.001$;

${ }^{c}$ Significant gender differences $(p<.05)$ using the Fisher $\mathrm{Z}$ transformation.

Table 3. Age-Corrected Correlations Between PPVT-R Standard Scores and Attention Scores.

scores only for boys. There were no significant associations between these measures and performance on the PPVT-R for girls. For both boys and girls, PPVT-R scores correlated significantly with number of categories in the WCST and scores on the Arithmetic and Digit Span subtests of the WISC-R.

Taken together, these findings indicate that the child's ability to detect infrequent targets and inhibit erroneous responses, as measured by the CPT, tends to improve with increasing verbal intelligence for both boys and girls. An improvement in the ability to encode numerical information and shift attentive focus is also observed with increasing verbal skills in both genders. In contrast, the association between intelligence and speed of processing differs between boys and girls. 
In order to determine if the relationship between attention and intelligence is consistent across intelligence levels, we compared the attentional scores of children who differed in their PPVT-R scores. The mean of PPVT-R standard scores in the present sample was almost one standard deviation below the standardization sample ( 87.2 vs. 100 with standard deviations of 16.8 and 15, respectively), with girls obtaining lower scores than boys ( 85.3 vs. $89.0, p<.05)$. The poorer performance of our sample in the PPVT-R is not surprising given that most of the children came from minority backgrounds, and minority children often obtain lower scores on this test (Alant \& Beukes, 1986; Vaughn-Cooke, 1983). Despite this difference in performance, PPVT-R standard scores conformed to a normal distribution for both boys and girls. Therefore, we divided the sample into four groups, with children in each group scoring within one standard deviation. The four groups consisted of: (1) children with PPVT-R standard scores below 70 (31 boys and 38 girls); (2) children with standard scores between 70 and 84 (59 boys and 75 girls); (3) children with standard scores between 85 and 99 (67 boys and 65 girls); and (4) children with PPVT-R scores of 100 or above (57 boys and 43 girls).

As expected given the findings of the correlation analyses, children in the four PPVT-R groups differed in their attention scores, but the results varied depending on the specific attention test. For example, the children's scores on the Coding subtest increased steadily from 8.6 in group 1 to 12.1 in group 4. Similar findings were obtained on the Arithmetic (7.5, 8.2, 9.7, and 11 , respectively in each group) and Digit Span (8.4, 9.0, 9.3, and 10.4, respectively) subtests of the WISC-R. These findings were not surprising given the strong correlation between the PPVT-R and the WISC-R.

Scores on the WCST also differed according to group membership, although only children with above average PPVT-R scores (Standard Score > 100; group 4) differed significantly from the other groups. Post hoc comparisons revealed that these children completed more categories than their peers (3.8 in group 4 vs. 2.7, 2.7, and 2.9 in groups 1,2, and 3, respectively) and made more correct responses and fewer perseverative errors. They did not differ from the other groups in the number of nonperseverative errors or failures to maintain set $(p>.10)$.

A different pattern of results emerged from analyses of the CPT data. Even though there was a significant main effect of PPVT-R group in all CPT measures, only children with deficient verbal capacities differed significantly from their peers. Children with PPVT-R scores below 70 made more commission errors than children with PPVT-R scores above 85 (groups 3 and 4) in all CPT versions; they also tended to make more omission errors, but this finding only reached statistical significance for the Degraded version. In fact, the difficulty of children with deficient verbal skills was always more pronounced in the Degraded CPT. The interaction between PPVT-R group and version of the Digit Cancellation task provided further support for the view that these children have more difficulty on tasks that have a higher processing requirement. Compared to children of average verbal skills, children with deficient verbal capacities (PPVT-R $<70)$ made more omission errors and had slower completion times in the Distraction condition of the Digit Cancellation task than in the Standard condition.

We failed to find any significant interactions between PPVT-R group and time on task on the CPT (first vs. second half of the task), suggesting that children of various intelligence levels did not differ in their ability to sustain their focus of attention over time.

\section{Gender and Verbal Intelligence}

Reaction time on the CPT showed a significant Gender x Verbal Intelligence interaction. For boys, reaction time improved steadily with increasing PPVT-R scores; a similar 
improvement was not observed in girls. There was also a significant interaction between gender and PPVT-R group for completion time on the Digit Cancellation tasks $(p<.0001)$. Consistent with the reaction time data, completion time on the Digit Cancellation task decreased with increasing PPVT-R scores in boys, but remained stable in girls. These findings are compatible with the lack of significant correlations between measures of speed and PPVT-R scores in girls.

\section{Discussion}

Previous studies have shown that attention comprises distinct cognitive processes and that various measures of attention are not interchangeable, but assess these processes differentially in children as well as in adults (Mirsky, 1987; Mirsky et al., 1991; Pogge, Stokes, \& Harvey, 1992; Wolf \& Halperin, 1987). In this study we sought to determine whether the child's gender and verbal intelligence have a distinct impact on these processes as measured neuropsychologically.

\section{The Role of Gender}

Gender differences in attentional performance generally favored girls. Girls had faster completion times and made fewer errors on the Digit Cancellation task. They also obtained higher scores on the Coding subtest of the WISC-R and made fewer commission errors on the CPT. These findings suggest that girls were more skillful at focusing their attention on a particular target, ignoring extraneous information, and executing a rapid response. Boys, in turn, had faster reaction times on the CPT and in some cases tended to adopt a more lenient response strategy.

Some of these gender differences, particularly those that reflect impulsivity or disinhibition (e.g., higher error rate, faster reaction times, and more lenient response criterion on the CPT), may reflect differences in maturation rate. Girls mature earlier than boys (Tanner, 1962), and variations in physical maturation are likely to be accompanied by changes in behavior and cognitive performance. Gender differences in activity level and impulsivity, for example, are in part mediated by maturation status (Eaton \& Piklai-Yu, 1989; Maccoby \& Jacklin, 1974). Maturity may also be playing a role in gender differences in cognitive impulsivity. Reports that younger children are faster and make more commission errors on the CPT than older children (Greenberg \& Waldman, 1993) are consistent with this hypothesis. Further evidence for the maturation hypothesis is provided by the results of a study by Gale and Lynn (1972). These investigators found that girls made fewer errors on a vigilance task at 7, 8, and 12 years of age, but did not differ from boys between 9 and 11 years. The particular ages at which girls performed better than boys coincide with 'spurts' in brain development (Epstein, 1974; Hudspeth \& Pribram, 1992) and, therefore, the girls' advantage at these ages may be secondary to their being further along the developmental path.

Other gender differences in attentional performance are likely to be more enduring and to reflect differences in brain organization. The brain is subject to the organizational effects of gonadal steroid hormones during sensitive periods in gestation or early postnatal life, and these effects influence gender dimorphic behaviors as well as cognitive performance. There are well-established differences between males and females in cognitive abilities. Males typically perform better on mathematical reasoning tasks and on tasks that require complex visual-spatial skills, whereas females excel on tests of verbal fluency, manual dexterity, and visual scanning (Gouchie \& Kimura, 1991; Kimura, 1992). Although gender differences in rearing practices and experience may contribute to variations in cognitive performance, the role of hormones appears to be substantial. For example, gender differences in cognitive abilities, particularly in 
visual-spatial skills, become more pronounced at puberty (Petersen, 1976), suggesting that hormonal changes play a role in their ontogenesis. Further, these differences can be reversed or attenuated in conditions characterized by aberrant levels of gender hormones such as Turner Syndrome (Murphy et al., 1994) and adrenal hyperplasia (Hines \& Shipley, 1984; Resnick, Berenbaum, Gottesman, \& Bouchard, 1986), and are affected by fluctuations in hormonal levels during the menstrual cycle (Becker, Creutzfeldt, Schwibbe, \& Wuttke, 1982; Jensen, 1982).

Gender differences in perceptual speed and visual scanning may account for the girls' excellence in some of the attention tests, most notably, in the Coding subtest and the Digit Cancellation task. Both the Digit Cancellation task and the CPT require the subject to focus on relevant stimuli while simultaneously inhibiting irrelevant responses (Cooley \& Morris, 1990). We know from the CPT results that boys and girls do not differ in their ability to focus and detect infrequent targets, even in the more demanding versions of this task. Our findings also indicate that they were equally proficient at ignoring distracting auditory information and at sustaining a focus of attention over time. Yet, these tasks differ in important ways. On the CPT, for example, the stimuli are presented individually, whereas on the Digit Cancellation task and the Coding subtest, the child is required to scan a visual array rapidly and make a response. Therefore, it is possible that girls' superiority on these tasks is secondary to their superior perceptual speed and visual scanning abilities.

\section{The Role of Intelligence}

Our findings indicate that verbal intelligence has an impact on attention, but that this effect is not consistent across levels of performance or attention processes. For example, the child's ability to encode information, as measured by the Arithmetic and Digit Span subtests of the WISC-R, improves steadily with increasing PPVT-R scores. A similar trend was not observed in the capacity to shift attentional focus, as assessed by the WCST. On this test, only children with above average verbal skills (PPVT-R scores $>100$ ) showed a beneficial effect of these skills in their performance on this task. Performance on the WCST is mediated by parts of the frontal cortex (Milner, 1963; Robinson, Heaton, Lehman, \& Stilson, 1980) which are not fully matured until late adolescence or early adulthood (Hudspeth \& Pribram, 1992; Yakovlev \& LeCours, 1967). The importance of maturation may be especially pronounced in this age group because these children may vary considerably in the functional maturity of their frontal cortices, and only a limited number of children may possess the maturity or skills necessary to perform adequately on this task.

In contrast, we found that children with verbal intelligence ranging from the low average to the very superior range did not differ significantly in their ability to sustain their focus of attention and inhibit impulsive responses on the CPT. In fact, only children with deficient verbal intelligence (i.e., PPVT-R scores $<70$ ) performed worse than their peers. This finding sheds some light into the lack of association between intelligence and performance on vigilance tasks in children with average cognitive capacities (Halperin et al., 1991) and the lower performance of children with mental retardation on these tasks (Warm \& Berch, 1985).

In addition, we did not find a strong association between intelligence level and ability to ignore extraneous information. Children with low verbal intelligence were not found to be more distractible or susceptible to irrelevant information. Similarly, these children did not differ in their ability to sustain their focus of attention over time. These results do not support Stankov's (1989) hypothesis that individuals with higher IQs would show a stronger vigilance decrement because they lose interest in monotonous tasks more easily than individuals with low IQs. These findings are also inconsistent with the results reported by Hagen and Huntsman (1971). These 
investigators found that children with mental retardation had more difficulty attending to relevant stimuli and ignoring irrelevant information than children with average intelligence. However, in Hagen and Huntsman's study only institutionalized children with mental retardation differed significantly from controls, whereas children with mental retardation residing at home (who are most likely similar to the present group) did not.

Consistent with Carter and Swanson's (1995) interpretation, our results indicate that children with poor verbal skills suggestive of mental retardation have limitations in their information processing capacities beyond their attention problems. Stated in another way, children with limited cognitive skills do not appear impaired visà-vis normal children on tasks requiring sustained or focused attention so long as the processing requirements of the tasks are not excessive. This notion was supported by the relative impairment these children exhibited on the Degraded CPT and on the Distraction version of the Digit Cancellation task. Therefore, tasks with greater processing demands (Schneider \& Shiffrin, 1977; Shiffrin \& Schneider, 1977) affected these children differentially, and appeared to be more sensitive to the effects of cognitive impairment.

\section{The Interaction of Gender and Intelligence}

Given that boys, as a group, are more active and impulsive than girls, some investigators have suggested that these behaviors may interfere with their cognitive performance (Maccoby, 1967; Maccoby \& Jacklin, 1974). In other words, impulsivity and overactivity are negatively associated with intelligence only for boys. Our findings, however, did not support this hypothesis. For example, although the correlation between verbal intelligence and number of commission errors in the CPT, commonly thought to reflect impulsivity, was stronger for boys, both genderes showed the same pattern of performance. Furthermore, measures of speed showed the opposite relationship. Speed of processing was not correlated with verbal intelligence for girls, but was positively correlated for boys. These findings suggest that cognitive impulsivity is distinguishable from rate of responding, and that only impulsivity is associated with lower cognitive performance.

\section{Implications for Clinical Practice}

The present results emphasize the impact of gender and intelligence on attentional performance, and may have important implications for clinical practice.

Although children with mental retardation tend to do worse on attention tests than children with normal IQs (e.g., Hagen \& Huntsman; 1971; Rosvold et al., 1956; Warm \& Berch, 1985), our results suggest that these children may have particular difficulties only on tasks with high processing demands. As a result, simple attention tasks can be developed to diagnose specific attention deficits in this population. The importance of devising appropriate assessment procedures to identify attention deficits in children with mental retardation is emphasized by the positive response that these children exhibit to a variety of psychopharmacological interventions (Dickerson-Mayes, Crites, Bixler, Humphrey, \& Mattison, 1994; Payton, Burkhart, Hersen, \& Helsel, 1988).

Our results also suggest that using the same norms to identify boys and girls with attention problems would likely result in a greater number of false negative diagnoses among girls, and that the lower prevalence of attention disorders in girls may be an artifact of our diagnostic procedures. Several investigators have proposed that girls may not be identified as having $\mathrm{AD} / \mathrm{HD}$ as often as boys because they exhibit fewer behavioral problems (Berry et al., 1985; Brown et al., 1991). The present results suggest, further, that girls may not be identified as often 
as boys because they perform better on objective measures of attention. Studies of children referred for AD/HD either report no gender differences in attention (Breen, 1989; Brown et al., 1991; Horn, Wagner, \& Ialongo, 1989) or find more cognitive and learning problems in the girls (Berry et al., 1985; James \& Taylor, 1990). Given that girls generally perform better on attention tests, it is possible that we are diagnosing with $\mathrm{AD} / \mathrm{HD}$ only those girls with severe difficulties, and that, moreover, more girls may benefit from treatment than are currently being treated.

One could argue that by identifying girls that are impaired in attentional performance relative to their same-gender peers, we are in fact selecting a group of girls that do not have any significant problems. However, our analyses do not support this notion. We have found that girls who obtain lower scores on the attention tests also perform poorer academically, have lower verbal intelligence, and are rated by their teachers as having more maladaptive behaviors than their same-gender peers (Anthony et al., 1997).

\section{Relation of Attention to Brain Organization and Development}

In prior publications, some of us (Mirsky, 1987; Mirsky et al., 1991) have speculated that the attention system in the brain is a complex system, paralleling the complexity of the behavioral functions encompassed within the rubric of "attention". According to this formulation, different attentional functions are supported by distinct cerebral regions, which are nevertheless integrated into a single, functional entity. For example, neural elements in the thalamic and upper brainstem portions of the attention system support the vigilance or sustain attention function tapped by the CPT. Similarly, arithmetic or digit span recall, identified as the encoding function of attention is mediated by limbic structures such as the amygdala and hippocampus, and the capacity-to-shift the focus of attention depends on the integrity of parts of the dorsolateral prefrontal cortex.

Some of the brain regions involved in the support of attentional functions are not fully myelinated until adolescence (Hudspeth \& Pribram, 1992; Yakovlev \& LeCours, 1967). Prior research has shown that apparently identical cerebral lesions in immature animals and adults of the same species may have different effects on behavior, depending on whether the damaged structure has achieved adult form at the time of the lesion. Thus, Goldman $(1971,1974)$ reported that the effects of certain lesions in young animals were not detectable until the behavior dependent upon the structure in question would normally have been expected to appear. This formulation is also supported by the work of Epstein, who noted that, 'there is no catch-up in intellectual level, and the building of an adolescent brain on top of an architecturally abnormal brain yields an even greater abnormality' (Epstein, 1974, pp. 343-370). In the present context we note that the published reports of differences between children and adults in the effects of head trauma on attention (with less severe effects seen in children than in adults) may relate to the immaturity of certain regions of the child's brain at the time of the insult.

The implications of these studies for the present investigation are as follows: There may have been compromises to the brain of some of these children which may not be manifest until they reach adolescence. We have continued to assess this cohort of children into the middle school years in order to study the developmental trajectory of the several attentional processes and the continued impact of gender and intelligence on attention performance. These assessments will be the subject of subsequent reports.

\section{Acknowledgements}

We acknowledge the contributions of the City of Baltimore, its families and children, and the administration, faculty, and staff of the Baltimore City Public Schools. In particular we would like to thank Dr. 
Walter Amprey, Superintendent of Baltimore City Public Schools; Dr. Lillian Gonzales and Dr. Patsy Blacksheare, Deputy Superintendents; Dr. Juanita Lewis, Deputy Director; and the following Administrators who were, then, in the positions noted; Dr. Denise Borders, Chief of Accountability; Robert Solomon, Director, Special Education; Dr. Carla Ford, Specialist, Early Childhood Education; Louise Fink, Coordinator, Social Work Services; Dale Parker-Brown, Director, Compensatory Education; Matthew Riley, Director of the Eastern District; and Willie Foster, Director of Middle Schools. The studies on which this paper is based have been supported by the following grants, with supplements from the National Institute on Drug Abuse: National Institute of Mental Health (NIMH) Grant Number P50 MH38725, Epidemiologic Prevention Center for Early Risk Behavior; NIMH Grant Number 1R01 MH42968, Periodic Outcome of Two Preventive Trials; NIMH Grant Number 1R01 MH40859, Statistical Methods for Mental Health Preventive Trials.

Address correspondence to: Dr. Daisy Pascualvaca, Section on Clinical and Experimental

Neuropsychology, Laboratory of Brain and Cognition, National Institute of Mental Health, Building 15K, Room 103, Bethesda, Md 20892, USA.

Accepted for publication: November 1, 1996.

\section{References}

Alant, E., \& Beukes, S. M. (1986). The application of the Peabody Picture Vocabulary Test-Revised (PPVT-R) to non-mainstream children. South African Journal of Communication Disorders, 33, 7-14.

American Psychiatric Association (1994). Diagnostic and statistical manual of mental disorders (4th ed.). Washington, DC: Author.

Anthony, B. J., Rebok, G. W., Pascualvaca, D. M., Jensen, P., Ahearn, M. B., Kellam, S. G., \& Mirsky, A. F. (1997). Epidemiological investigation of attention performance in children. Relationships to classroom behavior and academic performance. Manuscript submitted for publication.

Becker, D., Creutzfeldt, O. D., Schwibbe, M., \& Wuttke, W. (1982). Changes in physiological, EEG and psychological parameters in women during the spontaneous menstrual cycle and following oral contraceptives. Psychoneuroendocrinology, 7, 75-90.

Berry, C. A., Shaywitz, S. E., \& Shaywitz, B. A. (1985). Girls with attention deficit disorder: A silent minority? A report on behavioral and cognitive characteristics. Pediatrics, 76, 801-809.

Breen, M. J. (1989). Cognitive and behavioral differences in ADHD boys and girls. Journal of Child Psychology, Psychiatry, and Allied Disciplines, 30, 711-716.

Brown, R. T., Madan-Swain, A., \& Baldwin, K. (1991). Gender differences in a clinic-referred sample of attention-deficit-disordered children. Child Psychiatry and Human Development, 22, 111-128.

Cantwell, D. P. (1975). Genetics and hyperactivity. Journal of Child Psychology and Psychiatry and Allied Disciplines, 16, 261-264.

Carter, J. D., \& Swanson, H. L. (1995). The relationship between intelligence and vigilance in children at risk. Journal of Abnormal Child Psychology, 23, 201-220.

Chelune, G. J., \& Baer, R. A. (1986). Developmental norms for the Wisconsin Card Sorting Test. Journal of Clinical and Experimental Neuropsychology, 8, 219-228.

Cooley, E. L., \& Morris, R. D. (1990). Attention in children: A neuropsychological based model for assessment. Developmental Neuropsychology, 6, 239-274.

Dickerson-Mayes, S., Crites, D. L., Bixler, E. O., Humphrey, F. J., \& Mattison, R. E. (1994). Methylphenidate and ADHD: Influence of age, IQ, and neurodevelopmental status. Developmental Medicine and Child Neurology, 36, 1099-1107.

Dolan, L., Kellam, S. G., Brown, C. H., Wertharmer-Larsson, L., Rebok, G., Mayer, L., Laudolff, J., Turkkan, J. S., Ford, C., \& Wheeler, L. (1993). The short-term impact of two classroom-based preventive interventions on aggressive and shy behaviors and poor achievement. Journal of Applied Developmental Psychology, 14, 317-345.

Dunn, L. M., \& Dunn, L. M. (1981). Peabody Picture Vocabulary Test-Revised. Circle Pines, MN: American Guidance Service.

Eaton, W. O., \& Piklai-Yu, A. (1989). Are gender differences in child motor activity level a function of gender differences in maturational status? Child Development, 60, 1005-1011.

Epstein, H. T. (1974). Phrenoblysis: Special brain and neural growth periods: Human mental development. Developmental Psychobiology, 7, 217-224. 
Gale, A., \& Lynn, R. (1972). A developmental study of attention. British Journal of Educational Psychology, 42, 260-266.

Goldman, P. S. (1971). Functional development of the prefrontal cortex in early life and the problem of neuronal plasticity. Experimental Neurology, 32, 366-387.

Goldman, P. S. (1974). Recovery of function after CNS lesions in infant monkeys. Neuroscience Research Progress Bulletin, 12, 217-222.

Gouchie, C., \& Kimura, D. (1991). The relationship between testosterone levels and cognitive ability patterns. Psychoneuroendocrinology, 16, 323-334.

Greenberg, L. M., \& Waldman, I. D. (1993). Developmental normative data on the test of variables of attention (T.O.V.A.). Journal of Child Psychology, Psychiatry and Allied Disciplines, 34, 19-30.

Grier, J. B. (1971). Nonparametric indexes for sensitivity and bias. Psychological Bulletin, 75, 424-429.

Hagen, J. W., \& Huntsman, N. J. (1971). Selective attention in mental retardates. Developmental Psychology, 5, 151-160.

Halperin, J. M., Sharma, V., Greenblatt, E., \& Schwartz, S. T. (1991). Assessment of the continuous performance test: Reliability and validity in a nonreferred sample. Psychological Assessment: A Journal of Consulting and Clinical Psychology, 3, 603-608.

Halperin, J. M., Wolf, L. E., Pascualvaca, D. M., Newcorn, J. H., Healey, J. M., O'Brien, J. D., Morganstein, A., \& Young, J. G. (1988). Differential assessment of attention and impulsivity in children. Journal of the American Academy of Child and Adolescent Psychiatry, 27, 326-329.

Heaton, R. K. (1981). The Wisconsin Card Sorting Test. Odessa: Psychological Assessment Resources.

Hines, M., \& Shipley, C. (1984). Prenatal exposure to Diethylstilbestrol (DES) and the development of genderual dimorphic cognitive abilities and cerebral lateralization. Developmental Psychology, 20, 81-94.

Horn, W. F., Wagner, A. E., \& Ialongo, N. (1989). Gender differences in school-aged children with pervasive attention deficit hyperactivity disorder. Journal of Abnormal Child Psychology, 17, 109-125.

Hudspeth, W. J., \& Pribram, K. H. (1992). Psychophysiological indices of cerebral maturation. International Journal of Psychophysiology, 12, 19-29.

James, A., \& Taylor, E. (1990). Gender differences in the hyperkinetic syndrome of childhood. Journal of Child Psychology, Psychiatry, and Allied Disciplines, 34, 437-446.

Jensen, B. K. (1982). Menstrual cycle effects on task performance examined in the context of stress research. Acta Psychologica, 50, 159-178.

Kaufman, A. S. (1975). Factor analysis of the WISC-R at 11 age levels between $61 / 2$ and 16 1/2 years. Journal of Consulting and Clinical Psychology, 43, 135-147.

Kaufman, A. S. (1979). Intelligent testing with the WISC-R, New York: John Wiley \& Sons.

Kellam, S.F., \& Rebok, G.W. (1992). Building etiological theory through developmental epidemiologically-based preventive intervention trials. In J. McCord \& R.E. Tremblay (Eds.), Preventing antisocial behavior: Intervention from birth through adolescence (pp. 162-195). New York: Guilford Press.

Kellam, S. G., Branch, J. D., Agrawal, K. C., \& Ensminger, M. E. (1975). Menial health and going to school: The Woodlawn program of assessment, early intervention and evaluation. Chicago: University of Chicago Press.

Kellam, S. G., Werthamer-Larsson, L., Dolan, L., Brown, C. H., Mayer, L., Rebok, G. W., Anthony, J. C., Laudolff, J., Edelsohn, G., \& Wheeler, L. (1991). Developmental epidemiologically based preventive trials: Baseline modeling of early target behaviors and depressive symptoms. American Journal of Community Psychology, 19, 563-584.

Kimura, D. (1992). Cognitive function: Gender differences and hormonal influences. Neuroscience, S2, 41 - 43.

Kirchner, G. L., \& Knopf, I. J. (1974). Differences in vigilance performance of second-grade children as related to gender and achievement. Child Development, 45, 490-495.

Levy, F. (1980). The development of sustained attention (vigilance) and inhibition in children: Some normative data. Journal of Child Psychology, Psychiatry, and Allied Disciplines, 21, 77-84.

Levy, F., \& Hobbes, G. (1979). The influences of social class and gender on sustained attention (vigilance) and motor inhibition in children. Australian and New Zealand Journal of Psychiatry, 13, 231-234.

Lifshitz, M., Kugelmass, S., \& Karov, M. (1985). Perceptual-motor and memory performance of high-risk children. Schizophrenia Bulletin, 11, 74-84.

Maccoby, E. E. (1967). The development of gender differences. London: Tavistock.

Maccoby, E. E., \& Jacklin, C. N. (1974). The psychology of gender differences. Stanford, CA: Stanford University 
Press.

McGee, R., Williams, S., \& Silva, P. A. (1985). Factor structure and correlates of ratings of inattention, hyperactivity, and antisocial behavior in a large sample of 9 year-old children from the general population. Journal of Consulting and Clinical Psychology, 53, 480-490.

Milner, B. (1963). Effects of different brain lesions on card sorting. Archives of Neurology, 9, 90-100.

Mirsky, A. F. (1987). Behavioral and psychophysiological markers of disordered attention. Environmental Health Perspectives, 74, 191-199.

Mirsky, A. F., Anthony, B. J., Duncan, C. C., Ahearn, M. B., \& Kellam, S. G. (1991). Analysis of the elements of attention: A neuropsychological approach. Neuropsychology Review, 2, 109-145.

Murphy, D. G. M., Allen, G., Haxby, J. V., Largay, K. A., Daly, E., White, B. J., Powell, C. M., \& Schapiro, M. B. (1994). The effects of gender steroids, and the X chromosome, on female brain function: A study of the neuropsychology of adult Turner Syndrome. Neuropsychologia, 32, 1309-1323.

Offord, D. R., Boyle, M. H., Szatmari, P., Rae-Grant, N., Links, P. S., Cadman, D. T., Byles, J. A., Crawford, J. W., Munroe Blum, H., Byrne, C., Thomas, H., \& Woodward, C. (1987). Ontario child health study: Six-month prevalence of disorder and rates of service utilization. Archives of General Psychiatry, 44, 832-836.

Payton, J. B., Burkhart, J. E., Hersen, M., \& Helsel, W. J. (1988). Treatment of ADHD in mentally retarded children: A preliminary study. Journal of the American Academy of Child and Adolescent Psychiatry, 28, 761-767.

Petersen, A. C. (1976). Physiological androgyny and cognitive function in adolescence. Developmental Psychology, 12, 524-533.

Pogge, O. L., Stokes, J., \& Harvey, P. D. (1992). Empirical evaluation of the neuropsychology of attention: A confirmatory factor analysis of the Mirsky model in adolescents inpatients. Biological Psychiatry, 31, 112A.

Rebok, G. W., Hawkins, W. E., Krener, P., Mayer, L. S., \& Kellam, S. G. (1996). Effect of concentration problems on the malleability of children's aggressive and shy behaviors. Journal of the American Academy of Child and Adolescent Psychiatry, 35, 193-203.

Resnick, S. M., Berenbaum, S. A., Gottesman, I.I., \& Bouchard, T. J. (1986). Early hormonal influences on cognitive functioning in Congenital Adrenal Hyperplasia. Developmental Psychology, 22, 191-198.

Robinson, A. L., Heaton, R. K., Lehman, R. A. W., \& Stilson, D. W. (1980). The utility of the Wisconsin Card Sorting Test in detecting and localizing frontal lobe lesions. Journal of Consulting and Clinical Psychology, 48, 605-614.

Rosvold, H. E., Mirsky, A. F., Sarason, I., Bransome, E. Jr., \& Beck, L. H. (1956). A continuous performance test of brain damage. Journal of Consulting Psychology, 20, 343-350.

Rowe, K. J., \& Rowe, K. S. (1992). The relationship between inattentiveness in the classroom and reading achievement (Part B): An explanatory study. Journal of the American Academy of Child and Adolescent Psychiatry, 31, 357-368.

Rutter, M., Tizard, J., \& Whitmore, K. (1970). Education, health and behavior. London: Longman.

Schneider, W., \& Shiffrin, R. M. (1977). Controlled and automatic information processing: I. Detection, search and attention. Psychological Review, 81, 1-66.

Shiffrin, R. M., \& Schneider, W. (1977). Controlled and automatic human information processing: II. Perceptual learning, automatic attending, and a general theory. Psychological Review, 87, 127-190.

Stankov, L. (1989). Attentional resources and intelligence: A disappearing link. Personality and Individual Differences, 10, 957-968.

Tanner, J. M. (1962). Growth at adolescence. Oxford: Blackwell Scientific Publications.

Vaughn-Cooke, B. F. (1983). Improving language assessment in minority children. American Speech and Hearing Association, September, 29-33.

Warm, J. S., \& Berch, D. B. (1985). Sustained attention in the mentally retarded: International Review of Research in Mental Retardation, 13, 28-33.

Weber, A. M., \& Segalowitz., S. J. (1990). A measure of children's attentional capacity. Developmental Neuropsychology, 6, 13-23.

Wechsler, D. (1974). Wechsler Intelligence Scale for Children-Revised. New York: Psychological Corporation.

Wolf, L. E., \& Halperin, J. M. (1987). Assessment of attention in psychiatrically disturbed children: What are we really measuring? Journal of Clinical and Experimental Neuropsychology, 9, 61.

Yakovlev, P. I., \& LeCours, A. R. (1967). The mylogenetic cycles of regional maturation of the brain. In A. Minkowski (Ed.), Regional development of the brain in early life (pp. 3-70). Oxford, England: Blackwell. 